\title{
Pilot study of the radio-emitting AGN population: the emerging new class of FR 0 radio-galaxies
}

\author{
Ranieri D. Baldi ${ }^{1,2,3}$, Alessandro Capetti ${ }^{4}$, and Gabriele Giovannini ${ }^{5,6}$ \\ 1 SISSA-ISAS, via Bonomea 265, 34136 Trieste, Italy \\ e-mail: baldi@ph.technion.ac.il \\ 2 Physics Department, The Technion, 32000 Haifa, Israel \\ 3 Physics Department, Faculty of Natural Sciences, University of Haifa, 31905 Haifa, Israel \\ ${ }^{4}$ INAF-Osservatorio Astrofisico di Torino, Strada Osservatorio 20, 10025 Pino Torinese, Italy \\ 5 Dipartimento di Fisica e Astronomia, Università di Bologna, via Ranzani 1, 40127 Bologna, Italy \\ ${ }^{6}$ INAF-Istituto di Radio Astronomia, via P. Gobetti 101, 40129 Bologna, Italy
}

Received 28 November 2014 / Accepted 28 January 2015

\begin{abstract}
We present the results of a pilot JVLA project aimed at studying the bulk of the radio-emitting AGN population, that was unveiled by the NVSS/FIRST and SDSS surveys. The key questions are related to the origin of their radio-emission and to its connection with the properties of their hosts. We obtained A-array observations at the JVLA at 1.4, 4.5, and 7.5 GHz for 12 sources, a small but representative subsample. The radio maps reveal compact unresolved or only slightly resolved radio structures on a scale of $1-3 \mathrm{kpc}$, with the one exception of a hybrid FR I/FR II source extended over $\sim 40 \mathrm{kpc}$. Thanks to either the new high-resolution maps or to the radio spectra, we isolated the radio core component in most of them. We split the sample into two groups. Four sources have low black hole (BH) masses (mostly $\sim 10^{7} M_{\odot}$ ) and are hosted by blue galaxies, often showing evidence of a contamination from star formation to their radio emission, and are associated with radio-quiet (RQ) AGN. The second group consists in seven radio-loud (RL) AGN, which are located in red massive $\left(\sim 10^{11} M_{\odot}\right)$ early-type galaxies, have high BH masses $\left(\gtrsim 10^{8} M_{\odot}\right)$, and are spectroscopically classified as low excitation galaxies (LEG). These are all characteristics typical of FR I radio galaxies. They also lie on the correlation between radio core power and [O III] line luminosity defined by FR Is. However, they are more core-dominated (by a factor of $\sim 30$ ) than FR Is and show a deficit of extended radio emission. We dub these sources "FR 0" to emphasize their lack of prominent extended radio emission, which is their single distinguishing feature with respect to FR Is. The differences in radio properties between FR 0s and FR Is might be ascribed to an evolutionary effect, with the FR 0 sources undergoing rapid intermittency that prevents the growth of large-scale structures. However, this contrasts with the scenario in which low-luminosity radio-galaxies are fed by continuous accretion of gas from their hot coronae. In our preferred scenario the lack of extended radio emission in FR 0s is due to their lower jet Lorentz $\Gamma$ factor with respect to FR Is. The slower jets in FR 0s are more subject to instabilities and entrainment, which causes their premature disruption.
\end{abstract}

Key words. galaxies: active - galaxies: elliptical and lenticular, cD - galaxies: nuclei - galaxies: jets - radio continuum: galaxies

\section{Introduction}

The advent of large-area surveys with high sensitivity and the cross-match of radio and optical data opened the possibility of using large samples of extragalactic sources to investigate the links between the radio properties, the central engine, and the host galaxies. Based on the recent results of Baldi \& Capetti (2009) and Baldi \& Capetti (2010), a new population of radioemitting sources turns out to dominate in the local Universe.

Best et al. (2005) built a sample of 2215 radio-galaxies (RGs) by cross-correlating the SDSS (DR2), NVSS, and FIRST datasets (hereafter the SDSS/NVSS sample). This sample is selected at $F_{1.4}>5 \mathrm{mJy}$ and includes RGs up to $z \sim 0.3$, covering the range of radio luminosity $L_{1.4} \sim 10^{22}-10^{26} \mathrm{~W} \mathrm{~Hz}^{-1}$. All morphologies are represented, including twin-jets and corejet FR Is, narrow- and wide-angle tails, and FR IIs. However, most of them $(\sim 80 \%)$ are unresolved or barely resolved at the 5" FIRST resolution.

The SDSS/NVSS sample is mostly ( 90\%) composed of radio-loud active galactic nuclei (RL AGN), which represent the bulk of the RG population (with a space density $>100$ times higher than $3 \mathrm{C}$ sources) but it is virtually unexplored. A few partial results have been already obtained, even though the radio data available are extremely limited, generally only FIRST and NVSS images with angular resolution problems and the missing radio spectral coverage (they are generally too faint to be detected in the existing high-frequency surveys). In a previous paper, Baldi \& Capetti (2010) found the following results on this sample:

1) The AGN power estimated from the optical line luminosity of the SDSS/NVSS sample is at the same level of classical FR I RGs. However, the sample shows a deficit of a factor $\sim 100$ of radio emission with respect to the $3 \mathrm{C}$ sources with the same optical power.

2) The SDSS/NVSS sample generally shows optical emission line ratios typical of AGN: most of them $(\sim 70 \%)$ can be classified as low excitation galaxies (LEG) similarly to the FR Is, but a substantial fraction of high excitation galaxies (HEG) is also present ${ }^{1}$.

1 We bear in mind that HEG galaxies in the $3 \mathrm{C}$ catalog are all FR II RGs (Buttiglione et al. 2010). 
3) Most of the hosts of the SDSS/NVSS are statistically indistinguishable from those of $3 \mathrm{C}$ sources from the point of view of morphology, color, stellar, and black hole masses. They are generally associated with giant elliptical galaxies with no signs of star formation and are located in dense environment. Thus the deficit in radio emission cannot be ascribed to differences in their hosts.

4) A small fraction $(\sim 10 \%)$ of the sample shows rather different photometric properties and represents the radioquiet (RQ) AGN contamination to the main population of RL AGN.

These results have recently been confirmed by the analysis of a larger sample, obtained from the SDSS DR7, by Best \& Heckman (2012).

Similar results to the analysis of the SDSS/NVSS sample come from a parallel study of a sample of 14 nearby giant early-type galaxies (ETGs) of extremely low radio luminosity $\left(10^{20-22} \mathrm{~W} \mathrm{~Hz}^{-1}\right.$ at $1.4 \mathrm{GHz}$; Baldi \& Capetti 2009). They are named CoreG and are much fainter than FR Is (Balmaverde $\&$ Capetti 2006). Despite the low radio power, the CoreG host genuine "miniature" RL nuclei, that are indistinguishable from the FR Is. In fact they extend the FR I relations at different wavelengths (Chiaberge et al. 1999; Balmaverde et al. 2006; Balmaverde \& Capetti 2006; Baldi et al. 2010), indicating a common synchrotron origin of the nuclear emission. However, CoreG show a less extended radio structure and a core dominance up to $\sim 100$ times higher than FR Is, but similar to the SDSS/NVSS sample. A high core dominance is generally interpreted as evidence of Doppler boosting in a radio source oriented at a small angle with respect to the line of sight. The correlation found with the core radio power and emission line luminosity (independent of orientation) indicates that this is a genuine deficit of extended radio emission and that no geometric effect is present.

However, the differences between CoreG and FR Is might be driven by their much lower radio luminosity. For this reason, a high-resolution radio study of the SDSS/NVSS sample, objects sharing the same range of power of FR Is, is required. In fact, the radio properties of the bulk of the RL population are virtually unexplored. and we have little or no information on their radio morphology or spectral properties. This severely hampers our ability to understand their nature and how they are related, on the one hand, to classical RGs and, on the other hand, to low-luminosity AGN. An improvement of the radio data is needed to provide a more complete view of the radio emission phenomenon.

We then undertook a project aimed at understanding the nature of the radio emission of such objects and its connection with the host galaxies. More precisely, we present new highresolution observations with the JVLA of a pilot sample of 12 sources selected from the SDSS/NVSS sample. We obtained A array observations in $L$ and $C$ bands, reaching a resolution of 0.2 . In particular, the $C$ band provides the possibility of measuring the high-frequency core emission, which is crucial for our purposes.

We aim at answering the following questions:

1) Which is the nature of the radio emission: star formation, radio outflows as in RQ AGN, or genuine emission from a relativistic jet as in RL AGN?

2) After we isolate the RL AGN, which is their level of core dominance? Are they highly core-dominated similarly to the CoreG, but unlike the 3C sources? Or, alternatively, are they just compact, but with well-developed extended radio structures that produce most of the radio emission?
3) Do they follow the relation between radio core and line emission defined by $3 \mathrm{C} / \mathrm{FR}$ Is? The new observations aim at isolating their genuine radio core emission, an essential information to test their nuclear similarity with the FR Is.

4) Is their radio structure characterized by jet(s), diffuse plumes, or a double-lobed morphology? Are these jets one- or twosided? The distribution of jet sidedness will constrain their speed.

5) Which is the fraction of compact steep spectrum sources in the sample?

6) Is there a relationship between the radio properties and the optical spectroscopic classification, meaning, do HEG and LEG differ from the point of view of their radio structure?

The paper is organized as follows. In Sect. 2 we define the sample. Section 3 presents the new JVLA observations for 12 sources. In Sect. 4 we analyze the radio and spectrophotometric properties of the sample. We discuss the results in Sect. 5, presenting a new class of radio-sources, FR 0s (Sect. 5.1). The summary and conclusions to our findings are given in Sect. 6. We provide the notes on the radio properties of the sources that show extended morphology in the Appendix A.

\section{Sample}

The objects observed with the JVLA have been extracted from the SDSS/NVSS Best et al. sample adopting the following criteria:

- redshift $z<0.1$

- the main optical emission lines detected at least $5 \sigma$ significance (for the $\mathrm{H} \beta$ we relaxed this requirement to $3 \sigma$ ) to ensure a reliable spectral classification,

- equivalent width for the [O III] lines larger than $3 \AA$ and with a measurement error smaller than $1 \AA$; this ensures that the line emission is associated with an AGN and not with a stellar origin (Capetti \& Baldi 2011),

- declination in the range $-10<\delta<10$ in order to optimize the observing strategy.

This procedure returned 68 objects. Twelve of them are then randomly selected for the observations. In Table 1 we give the main properties of the observed objects.

We used the spectroscopic diagnostic diagrams defined by Buttiglione et al. (2010) for the 3CR sample to recognize the nature of their nuclear emission. These diagnostics are formed by pairs of nuclear emission line ratios to separate active nuclei from star-forming galaxies (e.g. Baldwin et al. 1981) and, furthermore, to separate AGN into branches of different excitation level, that is LEG and HEG. This separation is similar to that introduced by Kewley et al. (2006) as a diagnostic to distinguish among the RQ AGN population, LINERs and Seyferts (Heckman 1980).

From the point of view of their optical spectral classification, our sample covers the whole ionization range (Fig. 1), with eight objects located in the LEG area and two objects falling in in the HEG region. Two objects (ID 590 and 625) straddle the separation between the two classes; we classified 590 (625) as LEG (HEG) since in two out of three diagnostic ratios it is consistent with such a classification.

In Fig. 2 we show their location in a diagram comparing the FIRST radio and optical [O III]. All objects show a large deficit of radio emission when compared to the FR Is part of the $3 \mathrm{C}$ sample by a factor ranging from $\sim 30$ to $\sim 1000$. 
R. D. Baldi et al.: Pilot study of the radio-emitting AGN population: the emerging new class of FR 0 radio-galaxies

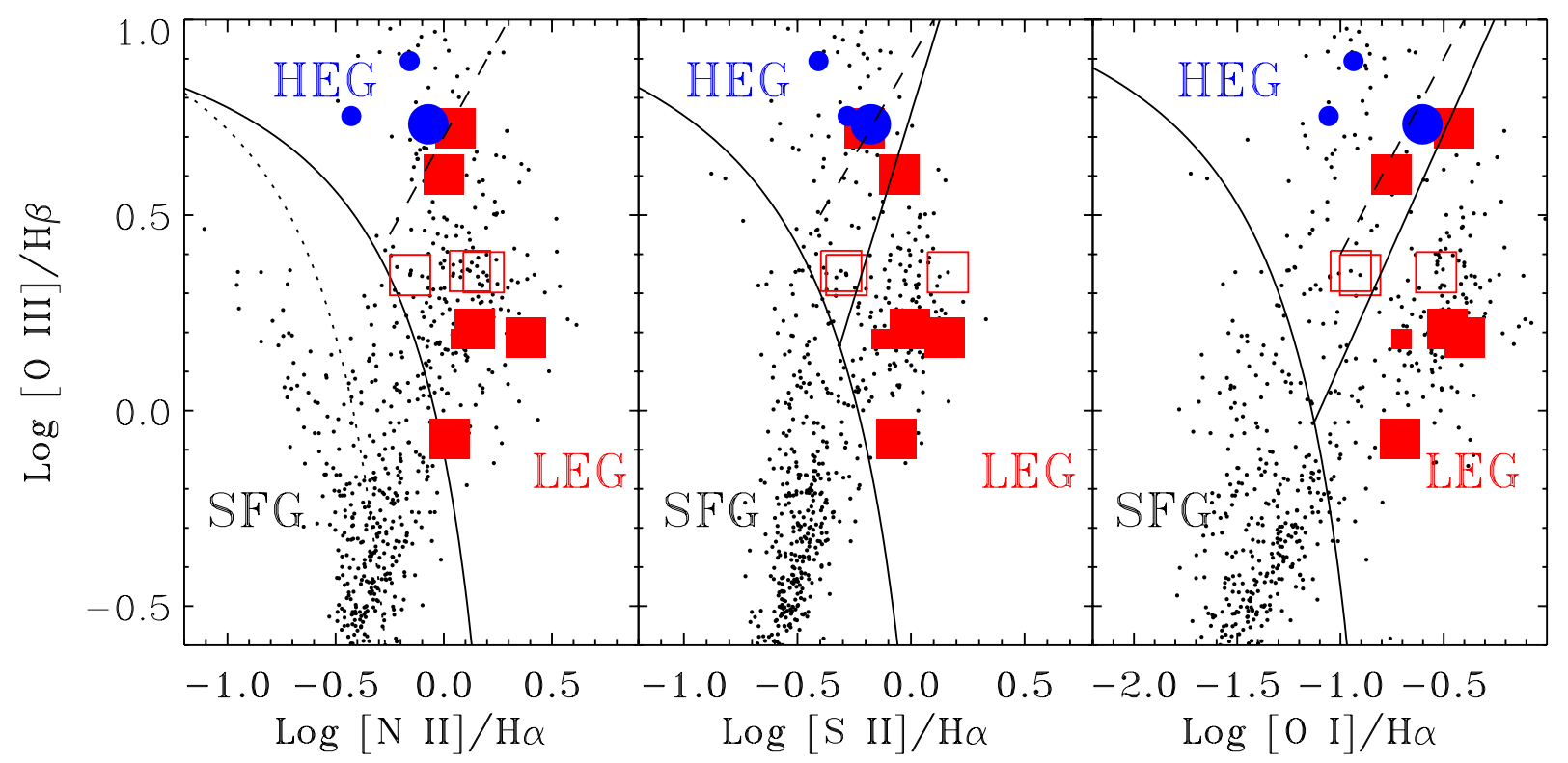

Fig. 1. Spectroscopic diagnostic diagrams for the galaxies of the sample. The small dot points correspond to the SDSS/NVSS sample studied by Baldi \& Capetti (2010), while the symbols in color represent galaxies with the new JVLA observations. The solid lines are from Kewley et al. (2006) and separate star-forming galaxies (SFG), LINER, and Seyfert. First panel: region between the two curves is populated by the composite galaxies. The dashed lines instead separate RL AGN into HEG from LEG (Buttiglione et al. 2010), which roughly correspond to the Seyfert and LINER classification for RL AGN. The red squares are the LEGs, while the blue circles are the HEGs. The large filled symbols are associated with BH masses $>10^{8} M_{\odot}$, large empty with $\sim 10^{7.7} M_{\odot}$, and small symbols with $<10^{7.3} M_{\odot}$.

Table 1. The sample.

\begin{tabular}{lcccccc}
\hline \hline ID & RA & Dec & $z$ & $F_{\text {NVSS }}$ & $F_{\text {FIRST }}$ & $F_{[\mathrm{O} \text { IIII] }}$ \\
\hline 511 & 353.1122 & -1.2017 & 0.094 & 3.2 & 1.57 & 92 \\
519 & 354.0168 & 0.0798 & 0.076 & 18.6 & 16.53 & 138 \\
524 & 356.5379 & 0.9858 & 0.093 & 24.8 & 25.92 & 43 \\
535 & 359.4337 & -0.1749 & 0.076 & 7.2 & 4.70 & 133 \\
537 & 0.4175 & 1.0920 & 0.061 & 6.5 & 6.49 & 38 \\
547 & 5.1446 & -0.4708 & 0.072 & 10.7 & 9.53 & 75 \\
567 & 9.9076 & -0.3288 & 0.055 & $<8.3$ & 2.86 & 148 \\
568 & 8.6813 & -0.0408 & 0.042 & 57.0 & 56.01 & 1161 \\
590 & 15.2546 & -0.4123 & 0.097 & 7.3 & 5.13 & 106 \\
605 & 18.8158 & 0.2135 & 0.045 & 42.4 & 46.52 & 81 \\
625 & 27.0677 & 0.3292 & 0.092 & 600.2 & 519.95 & 114 \\
656 & 43.3733 & -0.2349 & 0.029 & $<23.7$ & 6.96 & 1676 \\
\hline
\end{tabular}

Notes. Column description: (1) name; (2), (3) coordinates of the optical source; (4) redshift; (5), (6) radio flux at $1.4 \mathrm{GHz}$ from NVSS and FIRST surveys (mJy); (7) [O III] emission line flux $\left(10^{-17} \mathrm{erg} \mathrm{s}^{-1} \mathrm{~cm}^{-2}\right)$.

They are all (but one) located in the region of higher density of the SDSS/NVSS sample, just avoiding the lowest line luminosity, which is due to the third selection requirement. ID 656 is on the boundary of the region of RQ Seyferts. Only one object (ID 625) falls in a poorly populated area, being one of the objects of higher radio and line luminosity. In Fig. 2 we also stress the similarity of our sample to the CoreG sample in terms of $L_{\mathrm{FIRST}} / L_{[\mathrm{O} \text { III] }]}$ ratio.

Figure 3 shows the SDSS optical images of the galaxies of the sample. Most of the targets have an optical morphology similar to that of elliptical galaxies. Some, namely ID 519, 537, 547 and 568, show edge-on disks, while 567 shows spiral arms.

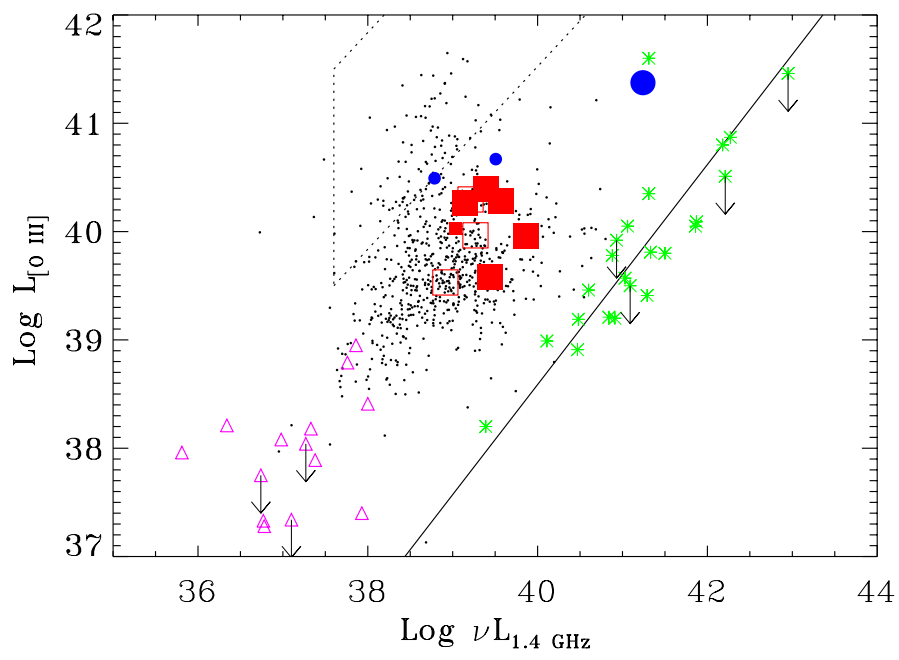

Fig. 2. FIRST vs. [O III] line luminosity $\left(\mathrm{erg} \mathrm{s}^{-1}\right)$. The colored points represent the 12 galaxies observed with the JVLA coded as in Fig. 1. The small dot points correspond to the SDSS/NVSS sample studied by Baldi \& Capetti (2010). The solid line represents the correlation between line and radio-luminosity derived for the 3CR/FR I sample. The dotted lines include the region where Seyfert galaxies are found. The empty pink triangles are the CoreG, and green stars the 3CR/FR Is.

\section{JVLA observations}

We obtained six hours of observations with the JVLA of NRAO ${ }^{2}$ with A array configuration on three days on December 2012 and January 2013. We observed 12 objects in scans of $2 \mathrm{~h}$ for each group composed of fours sources. Each source was observed for $\sim 20$ min spaced out by the pointing to the phase calibrators

2 The National Radio Astronomy Observatory is a facility of the National Science Foundation operated under cooperative agreement by Associated Universities, Inc. 

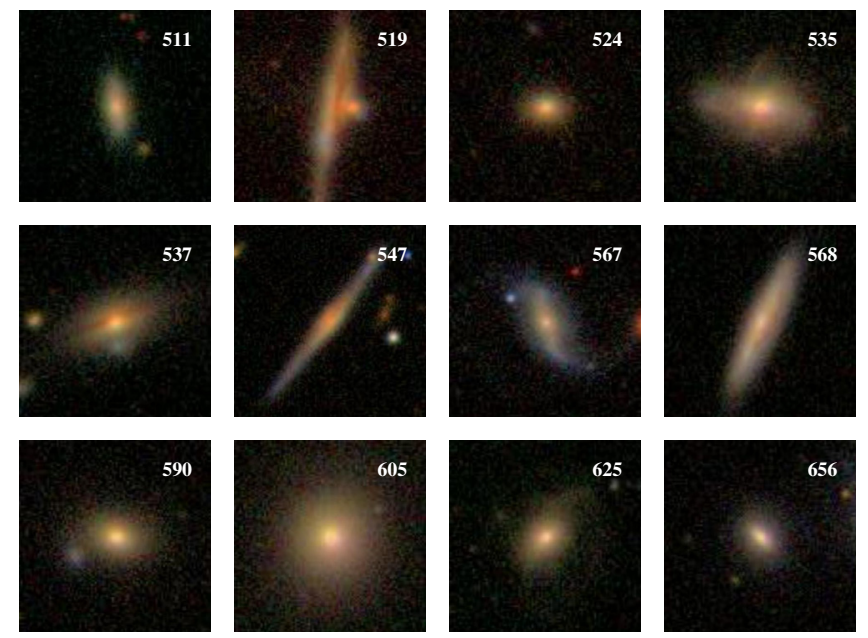

Fig. 3. SDSS optical images of the galaxies of our sample. The size of each frame is $40^{\prime \prime} \times 40^{\prime \prime}$.

(J0022+0014, J0059+0006, J0217+0144, and J2337-0230). The flux calibrator was $3 \mathrm{C} 48$ observed for $\sim 6-7 \mathrm{~min}$. The observations were performed in $L$ and $C$ bands; the exposure time was split into the two band configurations. While the $L$ band configuration corresponds to the default $1 \mathrm{GHz}$-wide band centered at $1.4 \mathrm{GHz}$, the $C$ band was modified to suit our purpose. We divided the available $2-\mathrm{GHz}$ bandwidth into two subbands of $1 \mathrm{GHz}$ centered at 4.5 and $7.5 \mathrm{GHz}$. This strategy allowed to obtain images in three different radio frequencies in two integration scans. Each of the three bands was configured into seven subbands of 64 channels of $1 \mathrm{MHz}$. The object ID 656 was only observed in $L$-band because of a telescope failure.

The data reduction was performed with the AIPS (Astronomical Image Processing System) package according to standard procedures. The final image was then produced from the calibrated data set by CLEANing to convergence. The final map was obtained using the task IMAGR with a beam size ranging between $\sim 0$.' 2 and $\sim 2$ '. 6 , according to the band, and different weights to increase the signal-to-noise ratio. We self-calibrated the maps of the sources with the gain flux $\gtrsim 10 \mathrm{mJy}$. The core component parameters were measured with the task JMFIT.

The noise level was measured in background regions near the target. The final noise is strongly affected (mainly in $L$ band) by interferences within the large bandwidth. At high frequency some observations show a gain error in a few telescopes that we were not able to recover in the calibration phase. Since in some sources the total flux density is too low to properly selfcalibrate the data in gain ( $\sim 10 \mathrm{mJy}$ or less) and even in phase (a few mJy or less), all these problems produced final images with a measured noise level higher than the expected thermal noise.

In Table 2 we collect the radio properties. In particular we give the total and core (when visible in the radio images) flux densities in the three observing bands.

\section{Results}

\subsection{Radio properties}

The FIRST radio maps of the selected objects show unresolved compact radio sources on a scale of $5^{\prime \prime}$, with the exception of ID 625 , which is extended on a scale of $\sim 35^{\prime \prime}$.

In the JVLA observations, most of the sources appear to be unresolved or only slightly resolved down to a resolution of $\sim 0$ ' 2 , which corresponds to $0.2-0.4 \mathrm{kpc}$. Some sources instead
Table 2. Results of the JVLA observations.

\begin{tabular}{|c|c|c|c|c|c|}
\hline ID & Morph. & $F_{\text {tot }}$ & $F_{\text {core }}$ & Beam & rms \\
\hline \multirow[t]{3}{*}{511} & SR & 1.15 & & $1^{\prime \prime} 2$ & 0.04 \\
\hline & $1^{\prime \prime}$ & 0.34 & 0.29 & 0.4 & 0.015 \\
\hline & $1.8 \mathrm{kpc}$ & 0.26 & 0.26 & 0.2 & 0.01 \\
\hline \multirow[t]{3}{*}{519} & SR & 12.21 & 9.4 & $1^{\prime \prime} .2$ & 0.05 \\
\hline & 0.9 & 3.8 & 2.92 & 0.4 & 0.04 \\
\hline & $1.3 \mathrm{kpc}$ & 2.2 & 1.96 & $0 ! 2$ & 0.013 \\
\hline \multirow[t]{3}{*}{524} & SR & 25.42 & & $1 . .2$ & 0.04 \\
\hline & 0.3 & 13.21 & & 0.4 & 0.05 \\
\hline & $0.5 \mathrm{kpc}$ & 9.16 & & 0.2 & 0.05 \\
\hline \multirow[t]{3}{*}{535} & $\mathrm{P}$ & 4.91 & & 1 1.'2 & 0.05 \\
\hline & & 2.24 & & 0.4 & 0.02 \\
\hline & $<0.3 \mathrm{kpc}$ & 1.86 & & 0.2 & 0.015 \\
\hline \multirow[t]{3}{*}{537} & $\mathrm{P}$ & 5.00 & & $2^{\prime \prime} .2 \times 1^{\prime \prime} .2$ & 0.07 \\
\hline & & 3.57 & & $0 . \prime 4$ & 0.1 \\
\hline & $<0.2 \mathrm{kpc}$ & 2.99 & 2.99 & 0.2 & 0.02 \\
\hline \multirow[t]{3}{*}{547} & twosid. jet & 12.22 & & $1^{\prime \prime \prime} 7 \times 11^{\prime \prime} 0$ & 0.05 \\
\hline & $2^{\prime \prime}$ & 4.30 & & $0.7 \times 0 . .5$ & 0.02 \\
\hline & $2.8 \mathrm{kpc}$ & 2.62 & 0.93 & 0.2 & 0.01 \\
\hline \multirow[t]{3}{*}{567} & $\mathrm{P}$ & 3.30 & & $2,0 \times 11^{\prime \prime} 0$ & 0.04 \\
\hline & & 0.85 & & 0.4 & 0.02 \\
\hline & $<0.2 \mathrm{kpc}$ & 0.74 & & 0.2 & 0.01 \\
\hline \multirow[t]{3}{*}{568} & double & 37.9 & & $2^{\prime \prime} 6 \times 2^{\prime \prime} .1$ & 0.04 \\
\hline & $1^{\prime \prime}$ & 27 & & 0.4 & 0.06 \\
\hline & $0.8 \mathrm{kpc}$ & 13.2 & & 0.35 & 0.02 \\
\hline \multirow[t]{3}{*}{590} & elongated & 5.9 & & $1^{\prime \prime} .5 \times 11^{\prime \prime} 1$ & 0.03 \\
\hline & 0.' 8 & 3.5 & & $0 . ' 4$ & 0.03 \\
\hline & $1.5 \mathrm{kpc}$ & 2.5 & & $0 . ' 25$ & 0.02 \\
\hline \multirow[t]{3}{*}{605} & $\mathrm{P}$ & 44.3 & & $2.1 \times 1^{\prime \prime} .3$ & 0.02 \\
\hline & & 42.1 & & $0 .{ }^{\prime} 6 \times 0.0^{\prime} 5$ & 0.02 \\
\hline & $<0.9 \mathrm{kpc}$ & 36.5 & & 0.25 & 0.04 \\
\hline \multirow[t]{3}{*}{625} & hybrid & 420 & & $1{ }^{\prime \prime} 1 \times 00^{\prime \prime} 9$ & 0.03 \\
\hline & $22^{\prime \prime}$ & 144 & 32.3 & $0 . \prime 6 \times 0.4$ & 0.03 \\
\hline & $37 \mathrm{kpc}$ & 103 & 31.5 & $0 . \prime 24 \times 0,21$ & 0.02 \\
\hline \multirow[t]{3}{*}{656} & SR & 7.4 & & $1{ }^{\prime \prime} 8 \times 11^{\prime \prime} 6$ & 0.06 \\
\hline & $0 . ' 8$ & - & - & - & - \\
\hline & $0.5 \mathrm{kpc}$ & - & - & - & - \\
\hline
\end{tabular}

Notes. Column description: (1) name; (2) radio morphology (P stands for point-source and SR for slightly resolved), size of the source in arcseconds and in kpc in the three following rows; (3) total radio flux (mJy) at $1.4 \mathrm{GHz}, 4.5 \mathrm{GHz}$ and $7.5 \mathrm{GHz}$ in the three following rows; (4) radio core flux (mJy) at $1.4 \mathrm{GHz}, 4.5 \mathrm{GHz}$ and $7.5 \mathrm{GHz}$ in the three following rows; (5) beam size (arcseconds) at $1.4 \mathrm{GHz}, 4.5 \mathrm{GHz}$ and $7.5 \mathrm{GHz}$ in the three following rows; (6) $\mathrm{rms}(\mathrm{mJy})$ at $1.4 \mathrm{GHz}, 4.5 \mathrm{GHz}$ and $7.5 \mathrm{GHz}$ in the three following rows.

show radio structures on a scale of $1-3 \mathrm{kpc}$ : ID 547 (a twin-jet source), ID 568 (a double source and lacking the radio core), and ID 590 (with an elongated radio morphology on a scale of 0.8 , possibly due to a bent two-sided jet structure). Finally, ID 625 shows a hybrid radio-structure (Gopal-Krishna \& Wiita 2000), being a FR I on the north side and a FR II on the south side on a scale of $\sim 40 \mathrm{kpc}$. Table 2 collects all the radio properties derived from the new observations. Appendix A provides additional comments on the radio maps for the sources that show extended structures (Figs. A.1 to A.4).

One of the main purposes of our program is to isolate the radio core component and to measure the core dominance of the sources of our sample. However, the high-resolution JVLA observations required with this aim and obtained with relatively short exposure time might be missing faint extended emission. To explore this possibility, we compared the JVLA $1.4 \mathrm{GHz}$ fluxes with those measured by FIRST and NVSS (see Table 3). The JVLA/FIRST flux ratios range from 0.71 to 1.28 , with an 
Table 3. Radio flux ratio.

\begin{tabular}{lcccc}
\hline \hline ID & $F_{\mathrm{FIRST}} / F_{\mathrm{NVSS}}$ & $F_{1.4 \mathrm{GHz}} / F_{\mathrm{NVSS}}$ & $F_{1.4 \mathrm{GHz}} / F_{\mathrm{FIRST}}$ & $R$ \\
\hline 511 & 0.49 & 0.36 & 0.73 & 0.08 \\
519 & 0.89 & 0.66 & 0.74 & 0.11 \\
524 & 1.05 & 1.02 & 0.98 & $<0.37$ \\
535 & 0.65 & 0.68 & 1.04 & 0.26 \\
537 & 1.00 & 0.77 & 0.77 & 0.46 \\
547 & 0.89 & 1.14 & 1.28 & 0.09 \\
567 & $-{ }^{a}$ & $-{ }^{a}$ & 1.15 & $0.26^{b}$ \\
568 & 0.98 & 0.66 & 0.71 & $<0.23$ \\
590 & 0.70 & 0.81 & 1.15 & 0.34 \\
605 & 1.10 & 1.04 & 0.95 & 0.86 \\
625 & 0.87 & 0.70 & 0.81 & 0.05 \\
656 & $-{ }^{a}$ & $-{ }^{a}$ & 1.06 & - \\
\hline
\end{tabular}

Notes. Column description: (1) name; (2) flux ratio between FIRST and NVSS; (3) ratio between 1.4-GHz flux from JVLA observation and NVSS flux; (4) ratio between 1.4-GHz flux from JVLA observation and FIRST flux; (5) core dominance $R$, i.e., ratio between 7.5-GHz core flux and NVSS flux. ${ }^{(a)}$ Two point-like FIRST sources are blended into a single NVSS catalog entry and ${ }^{(b)}$ the core dominance is estimated from the FIRST flux.

average value of 0.95 indicating that we recovered most of the radio emission. The comparison with the NVSS data is more complex because of the low resolution of this survey. Indeed, in two cases (ID 567 and ID 656) two point-like FIRST sources are blended into a single NVSS catalog entry. Leaving these two sources aside, the JVLA/NVSS flux ratios cover a range between 0.36 and 1.14 , with an average of 0.78 . We conclude that our measurements recover most of the total radio flux of our sources. In general, the fraction of missing flux is sufficiently small and does not to alter our view of the overall radio structure.

We obtained matched-beam radio maps to derive the radio spectra at the three frequencies (see Fig. 4). For the object ID 656 it is not possible to derive its spectrum because of the lack of its $C$-band observation.

In Fig. 4 we report the spectral slope $\alpha\left(F_{v} \propto v^{\alpha}\right)$, obtained between 1.4 and $4.5 \mathrm{GHz}$. The spectral indices range from -1.16 to -0.04 , which is indicative of both steep and flat spectra. We note that source 568 shows a strong steepening at $7.5 \mathrm{GHz}$. This might be due to some missing flux at the highest frequency, although we note that its double structures at 4.5 and $7.5 \mathrm{GHz}$ are very similar. We suggest that its spectra might be intrinsically steep.

One of the key points of this study is the measurement of the radio core. The high-resolution radio maps at $7.5-\mathrm{GHz}$ reveals a radio core component for five sources (ID 511, 519, 537, 547, and 625). For the remaining sources, we can use the radio spectra to measure the core emission. Two sources (ID 590 and 605) show an overall flat $(\alpha>-0.5)$ radio spectrum, consistent with a self-absorbed synchrotron emission; the flux at $7.5 \mathrm{GHz}$ is dominated by the radio core emission. For ID 535 and 567 a spectral flattening occurs at $7.5 \mathrm{GHz}$ where the core emerges over the optically thin radio emission. For the last three objects of the sample we used the total flux at $7.5 \mathrm{GHz}$ as upper limit for the radio core measurement.

After measuring the radio core component thanks to the new observations, we can include our sources in the $L_{\text {core }}$ Vs. $L_{[\mathrm{O} \text { III] }}$ plane (Fig. 5), similarly to what was done for CoreG in Baldi \& Capetti (2009). The radio core power distribution spans a range similar to the local 3CR/FR Is. Several sources lie in the same region as the 3CR/FR Is, also considering their line luminosities, but others show a radio core deficit (or line excess) with respect to the relation defined by the FR Is. The sources classified as LEGs, on average, lie closer to the relation than the HEGs.

The core dominance of our sources, $R$, is defined as the ratio between the source nuclear emission at $7.5 \mathrm{GHz}$ and the total flux density, for which we adopted the NVSS measurement (Table 3 ). We preferred to use the $7.5 \mathrm{GHz}$ core measurements, with respect to the usual definition based on $5 \mathrm{GHz}$ data, to limit the contamination from the extended structures as much as possible. The core dominance ranges between 0.05 and 0.86 . The lowest $R$ belongs to the most extended object, the FR I/FR II ID 625.

\subsection{Spectro-photometric properties}

We now explore the physical properties of the present objects based on the optical spectroscopic and photometric information available from the SDSS survey. We consider the mass of their central black hole (BH), the host mass and morphology, and the $D_{n}(4000)$ index (see Table 4), similarly to the study of SDSS/NVSS sample by Baldi \& Capetti (2010).

We estimated the $\mathrm{BH}$ masses $\left(M_{\mathrm{BH}}\right)$ from the stellar velocity dispersion adopting the relation of Tremaine et al. (2002). They range from $\sim 10^{7}$ to $\sim 10^{9} M_{\odot}$. The sources are associated with galaxies with a distribution of masses $^{3}$ in the range $10^{10}-10^{11.5} M_{\odot}\left(\right.$ and a median of $\left.\sim 10^{11} M_{\odot}\right)$.

We then studied the host type by using the concentration in$\operatorname{dex} C_{r}$ (defined as the ratio of the radii including $90 \%$ and $50 \%$ of the light in the $r$ band). ETGs have $C_{r} \geq 2.86$ (e.g. Strateva et al. 2001; Kauffmann et al. 2003; Bell et al. 2003). This cut basically corresponds to $\mathrm{E}$ type with a weak contamination of S0 and Sa (Bernardi et al. 2010). A less conservative selection of ETGs uses $C_{r} \geq 2.60$ (e.g., Nakamura et al. 2003; Shen et al. 2003), which yields a strong contribution from S0 and Sa.

The $4000 \AA$ break strength, $D_{n}(4000)$ (defined as the ratio between the fluxes in the wavelength range 3850-3950 $\AA$ and 4000-4100 $\AA$ ) is instead sensitive to the presence of a young stellar population (Balogh et al. 1999). Massive galaxies $\left(\log M_{*} / M_{\odot} \gtrsim 10.5\right)$ show a bimodal distribution of $D_{n}(4000)$ (Kauffmann et al. 2003). The transition between the two groups is located approximately at $D_{n}(4000) \sim 1.7$ with the red (blue) hosts having higher (lower) values.

A further diagnostic panel tool enables us to to qualitatively measure the amount of contamination in radio emission in the galaxy that is due to star formation. This method is based on the location of a galaxy in the $D_{n}(4000)$ versus $L_{1.4 \mathrm{GHz}} / M_{*}$ plane, where $M_{*}$ is the galaxy's stellar mass. Best et al. (2005) predicted the radio emission per unit of galaxy mass expected from a stellar population of a given age: when a galaxy shows an excess higher than 0.225 in $D_{n}(4000)$ above the curve corresponding to the prediction of a star formation event lasting $3 \mathrm{Gyr}$ and exponentially decaying, its radio emission is associated with the AGN.

The left panel of Fig. 6 shows the distribution of the objects in the spectro-photometric diagram composed of $C_{r}$ vs. $D_{n}(4000)$, while in the right panel we compare $L_{1.4 \mathrm{GHz}} / M_{*}$ and $D_{n}(4000)$. To explore the properties of the sample, we separated the sources into three groups based on the BH masses: high $>10^{8} M_{\odot}$, intermediate $\sim 10^{7.7} M_{\odot}$ and low $<10^{7.3} M_{\odot}$.

We note a different behavior of the sources in our sample that is related with their BH mass. A first group consists of four

$3 M_{*}$ is estimated from the $g$ and $z$ SDSS magnitudes following following Bell et al. (2007), which results in slightly different masses from those reported by Best et al. (2005). 

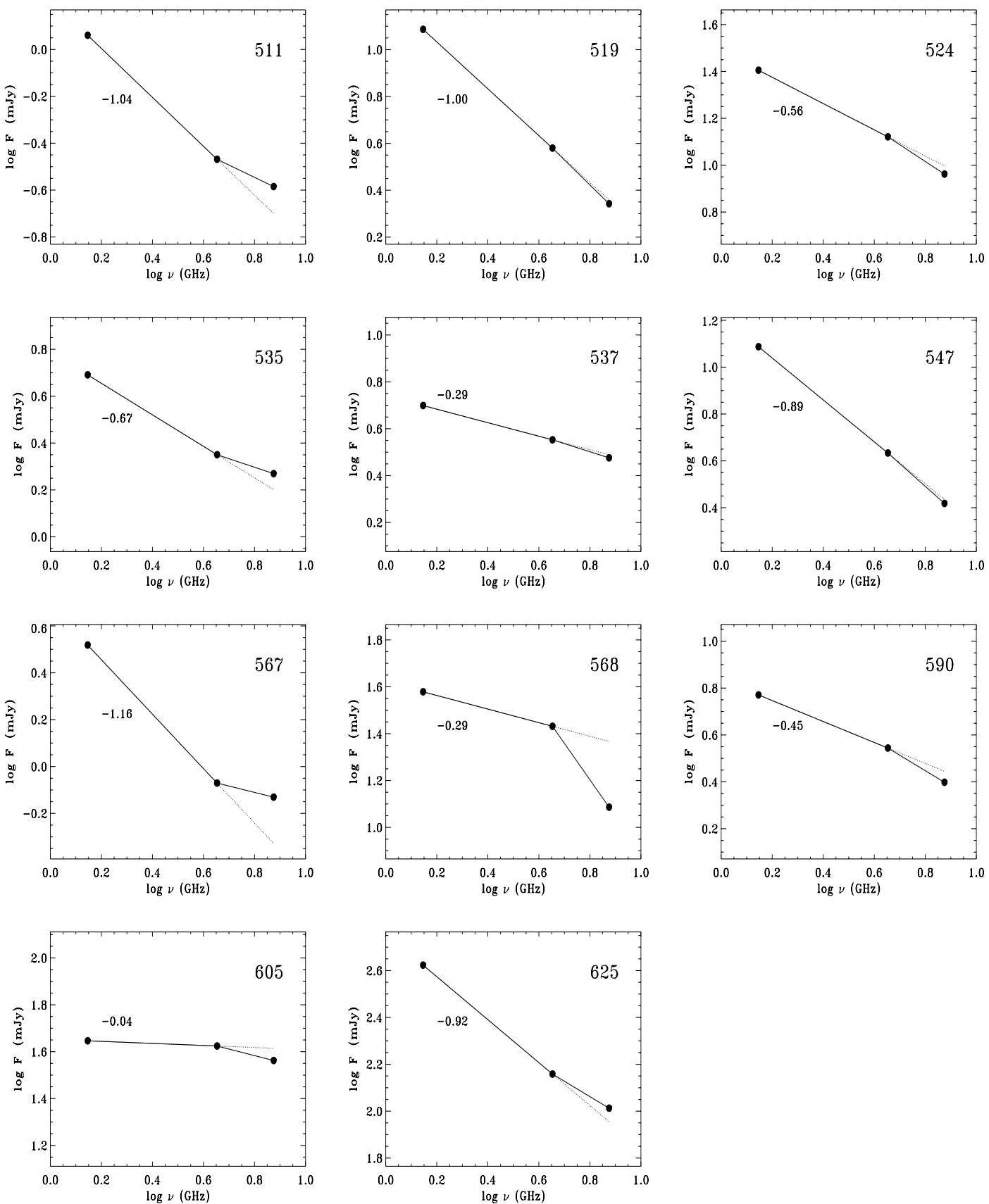

Fig. 4. Radio spectra of the 11 objects observed with JVLA observed at 1.4, 4.5 and $7.5 \mathrm{GHz}$. The dotted line is the extrapolation of the 1.4-4.5 GHz slope, which is reported in each panel.

sources with low (and one intermediate) BH masses, namely ID 511, 567, 568, and 656. Three of them (see Fig. 6, right panel), probably receive contribution of a star formation to their radio emission; this is also supported by their high NVSS/FIRST radio flux ratios, an indication of extended diffuse radio emission, that was missed by the FIRST images. The fourth object (ID 568) is consistent with being a Seyfert 2 galaxy, based on its location in Fig. 2 and on its spectrophotometric properties. They are all blue galaxies, having $D_{n}(4000)<1.6$. In the diagram comparing the core power, $L_{\text {core }}$, and emission line luminosity (Fig. 5) they all show a high deficit of radio core emission, typically of a factor of $\gtrsim 100$, with respect to the relation defined by $3 \mathrm{CR} / \mathrm{FR}$ Is.

Conversely, the second group includes sources with high/intermediate $\mathrm{BH}$ masses whose radio emission is dominated by the AGN. With the sole exception of ID 625 (the hybrid FR I/FRII radio source) they are associated with red massive ETGs belonging to the LEG spectroscopic class. With respect to the first group, they follow the $L_{\text {core }}$ Vs. $L_{[\mathrm{O} \text { III] }}$ relation more closely.

Figure 7 shows that the core dominance for this subsample ranges from $\log R \sim-1$ to $\sim 0$ with a mean value of $\sim-0.5$. This distribution is significantly different from that of 3CR/FRIs (with a $>99.9 \%$ probability, according to a Kolmogorov-Smirnoff test) while it is indistinguishable from the $R$ distribution of CoreG. We recall that we estimated $R$ as the ratio between the 7.5-GHz core emission and the total 1.4-GHz flux (NVSS), while for the 3CR/FRIs and CoreG we used the $5 \mathrm{GHz}$ core flux against the total 1.4-GHz flux (NVSS). However, since the radio core emission generally has a flat spectrum, this quantity 
R. D. Baldi et al.: Pilot study of the radio-emitting AGN population: the emerging new class of FR 0 radio-galaxies

Table 4. Spectroscopic and photometric properties.

\begin{tabular}{lccccccccccc}
\hline \hline ID & Opt. class & $D_{n}(4000)$ & $C_{r}$ & $M_{r}$ & $\log M_{*}$ & $\log M_{\mathrm{BH}}$ & $\log L_{[\mathrm{O} \text { III] }}$ & $\log L_{\mathrm{FIRST}}$ & $\log L_{\mathrm{NVSS}}$ & Log $L_{\text {core }}$ & Radio class \\
\hline 511 & LEG & 1.52 & 2.63 & -21.74 & 11.10 & 7.65 & 40.30 & 38.68 & 38.99 & 38.63 \\
519 & LEG & 1.74 & 2.42 & -22.11 & 11.30 & 8.67 & 40.28 & 39.50 & 39.56 & 39.31 & RQ \\
524 & LEG & 1.89 & 2.87 & -21.50 & 11.07 & 8.32 & 39.96 & 39.89 & 39.87 & $<40.16$ & FR 0 \\
535 & LEG & 1.75 & 2.83 & -22.61 & 11.48 & 8.76 & 40.26 & 38.96 & 39.14 & 39.29 & FR 0 \\
537 & LEG & 1.95 & 2.87 & -21.38 & 11.09 & 7.72 & 39.52 & 38.90 & 38.90 & 39.29 & FR 0 \\
547 & LEG & 1.67 & 3.57 & -21.62 & 11.18 & 7.64 & 39.97 & 39.22 & 39.27 & 38.93 & FR 0 \\
567 & LEG & 1.44 & 2.69 & -20.95 & 10.62 & 7.14 & 40.02 & 38.45 & $<38.91$ & 38.59 & RQ \\
568 & HEG & 1.56 & 2.33 & -20.99 & 10.76 & 7.19 & 40.67 & 39.50 & 39.51 & $<39.60$ & RQ \\
590 & LEG & 1.93 & 3.29 & -22.47 & 11.42 & 8.43 & 40.39 & 39.22 & 39.37 & 39.64 & FR 0 \\
605 & LEG & 1.99 & 3.10 & -21.86 & 11.15 & 8.57 & 39.57 & 39.47 & 39.44 & 40.10 & FR 0 \\
625 & HEG & 1.61 & 3.17 & -22.13 & 11.24 & 8.18 & 41.37 & 41.18 & 41.24 & 40.69 & FR I/FR II \\
656 & HEG & 1.39 & 3.16 & -19.25 & 9.97 & 7.08 & 40.50 & 38.26 & $<38.79$ & $<39.02$ & RQ \\
\hline
\end{tabular}

Notes. Column description: (1) name; (2) optical spectroscopic classification; (3) color based on $D_{n}$ (4000) (typical error 0.07-0.14); (4) concentration index (typical error 0.01-0.03); (5) $M_{r}$ absolute $r$-band magnitude (typical error <0.02); (6) galaxy stellar mass $M_{*}\left(M_{\odot}\right)$ (typical error 0.15); (7) black hole mass $M_{\mathrm{BH}}\left(M_{\odot}\right)$ (typical error 0.07-0.12); (8) [O III] luminosity (erg s ${ }^{-1}$; typical error 3-10\%); (9) 1.4 GHz FIRST luminosity $\left(\mathrm{erg} \mathrm{s}^{-1}\right.$ ); (10) $1.4 \mathrm{GHz}$ NVSS luminosity $\left(\mathrm{erg} \mathrm{s}^{-1}\right.$ ) used as total radio luminosity $L_{\mathrm{tot}}$; (11) $7.5 \mathrm{GHz}$ VLA luminosity (erg s${ }^{-1}$ ) used as radio core luminosity $L_{\text {core }}$; (12) radio class: radio-quiet (RQ) AGN, FR 0, hybrid FR I/FR II.

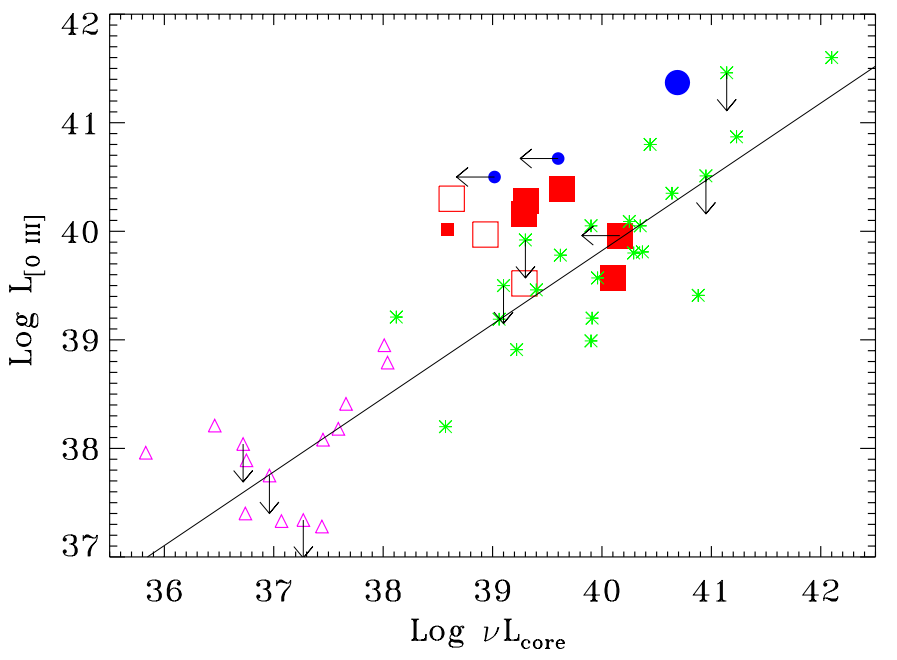

Fig. 5. Core radio power vs. [O III] line luminosity ( $\mathrm{erg} \mathrm{s}^{-1}$ ) for CoreG (pink triangles), 3CR/FR I radio-galaxies (green stars), and our sample (colored symbols like in Fig. 1). The line indicates the best linear fit the for $3 \mathrm{CR} / \mathrm{FR}$ Is.

is only weakly dependent on the frequency used for the core measurement, and this comparison is robust.

\section{Discussion}

The results discussed in the previous sections indicate that the twelve objects belonging to the SDSS/NVSS sample that were observed with the JVLA, can be divided into two main groups, that correspond to specific radio, spectroscopic, and photometric properties. Before discussing these groups in more detail, we treat here the only object that shows rather different properties. Not surprisingly, by exploring a sample of radio-sources, we found a classical extended RG, namely ID 625. As already mentioned, it has a hybrid FR I/FRII morphology extending over $\sim 40 \mathrm{kpc}$. All its characteristics are typical of FR II sources considering its $\mathrm{BH}$ mass, the blue host color, its spectroscopic class (HEG), and its location in both the $L_{\text {core }}$ and $L_{\text {tot }}$ vs. $L_{[\mathrm{O}}$ III] diagram in the region populated by the FR II part of 3C sample (Chiaberge et al. 2002; Buttiglione et al. 2010; Chiaberge \& Marconi 2011).
The first main group consists of four sources that are characterized by their low $\mathrm{BH}$ masses, mostly $\sim 10^{7} M_{\odot}$ and their blue color. Their radio and spectrophotometric properties indicate that they are RQ AGN (Table 4). The presence of an active nucleus is witnessed by their optical line ratios and equivalent widths, which are characteristic of AGN. Nonetheless, three of them show a substantial contamination from star formation to their radio emission. The fourth object (ID 568) shows a double radio morphology with a size of $0.8 \mathrm{kpc}$ and a steep spectrum, which is often observed in Seyfert galaxies (Edelson 1987; Nagar et al. 2005). In addition, its radio and line power locate this source in the area typical of the Seyfert galaxies. The contamination from RQ AGN ( $\sim 33 \%$ of the sample) is higher than observed in the whole SDSS/NVSS sample (Baldi \& Capetti 2010). This is because the selection criterion of this pilot sample based on the high [O III] equivalent width favors the inclusion of bright emission-line galaxies, which are preferentially RQ AGN.

The second group consists of seven sources, representing (together with the powerful source ID 625 discussed above) the genuine RL AGN population in the SDSS/NVSS sample. They are located in red massive $\left(\sim 10^{11} M_{\odot}\right)$ ETGs, have BH masses $\gtrsim 10^{8} M_{\odot}$ and are spectroscopically classified as LEGs (apart from the HEG-spectrum FR I/FR II 625). All these properties are shared with FR I RGs (e.g., Zirbel 1996; Cao \& Rawlings 2004; Floyd et al. 2008; Chiaberge et al. 1999; Baldi \& Capetti 2008, 2009; Buttiglione et al. 2010). Furthermore, their radio core and [O III] luminosities lie in the range typical of FR Is. Therefore, all the nuclear and host characteristics of this group are indistinguishable from those of FR Is. Nonetheless, the JVLA observations presented here show compact radio structures (with a limit to their size of $\sim 0.5 \mathrm{kpc}$ or, in a few cases, extending by at most $1-3 \mathrm{kpc}$ ) and lead to an estimate of their (average) core dominance a factor of $\sim 30$ higher than FR Is. The only feature distinguishing them from FR Is is then the substantial lack of extended radio emission. For this paucity we call them FR 0 in opposition to the jetted FR radio classes (Fanaroff \& Riley 1974).

\subsection{The FR 0 population}

The FR 0 classification corresponds to a combination of radio and spectro-photometric properties. In the radio band, FR 0s 

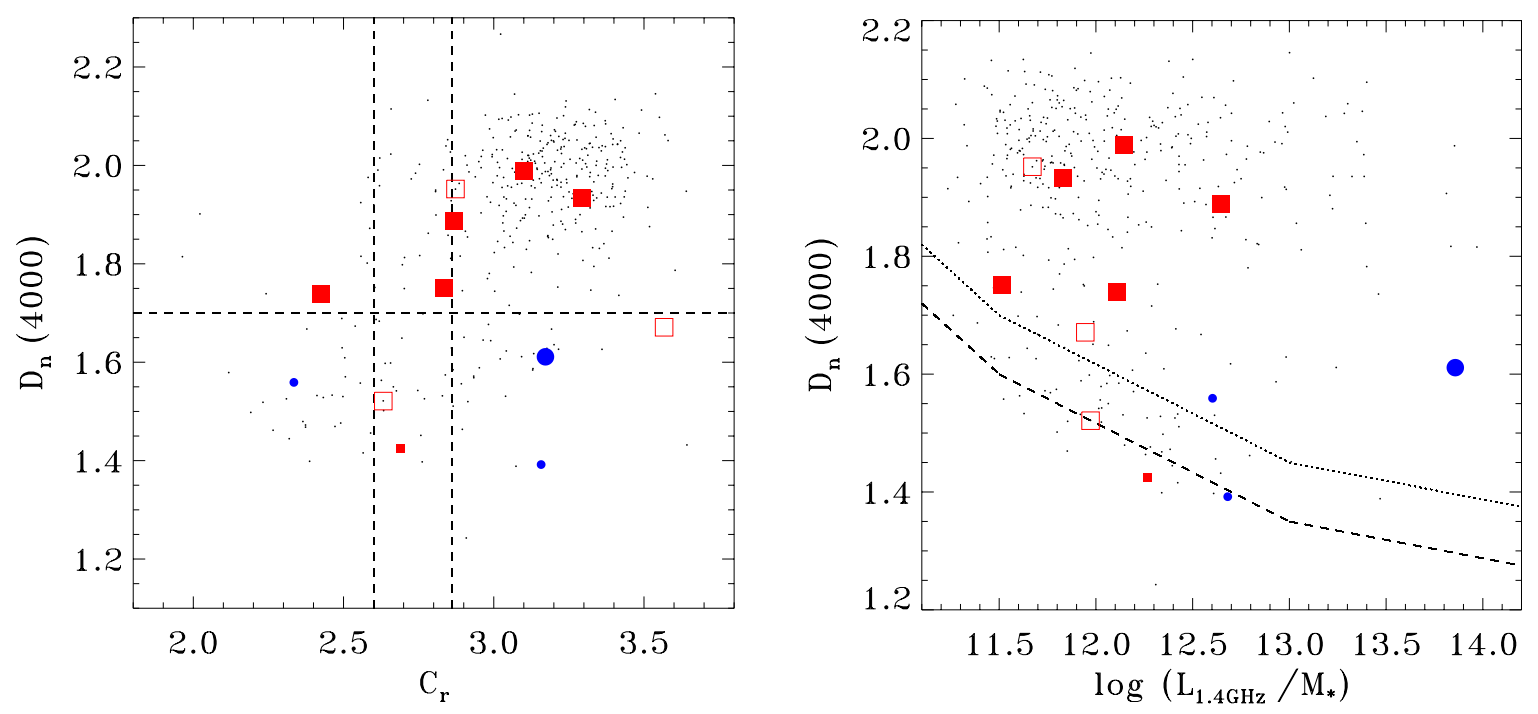

Fig. 6. Left panel: concentration index in the $r$ band, $C_{r}$, versus the $D_{n}(4000)$ index. The vertical lines correspond to the two definitions of ETGs $\left(C_{r}>2.86\right.$ or 2.60 , see text for details), while the horizontal line separates blue from red sources. Right panel: $D_{n}(4000)$ versus $L_{1.4 \mathrm{GHz}} / M_{*}$. The dashed curve is the empirical separation between the star-forming and AGN radio-emitting sources performed by Best et al. (2005; read the text for details). The dotted curve is shifted by 0.1 above in $D_{n}(4000)$ of the dashed curve to separate the objects with a possible star-formation contribution to their radio emission. The small dot points correspond to the SDSS/NVSS sample studied by Baldi \& Capetti (2010). The different color/shape symbols correspond to the different classes like in Fig. 1.
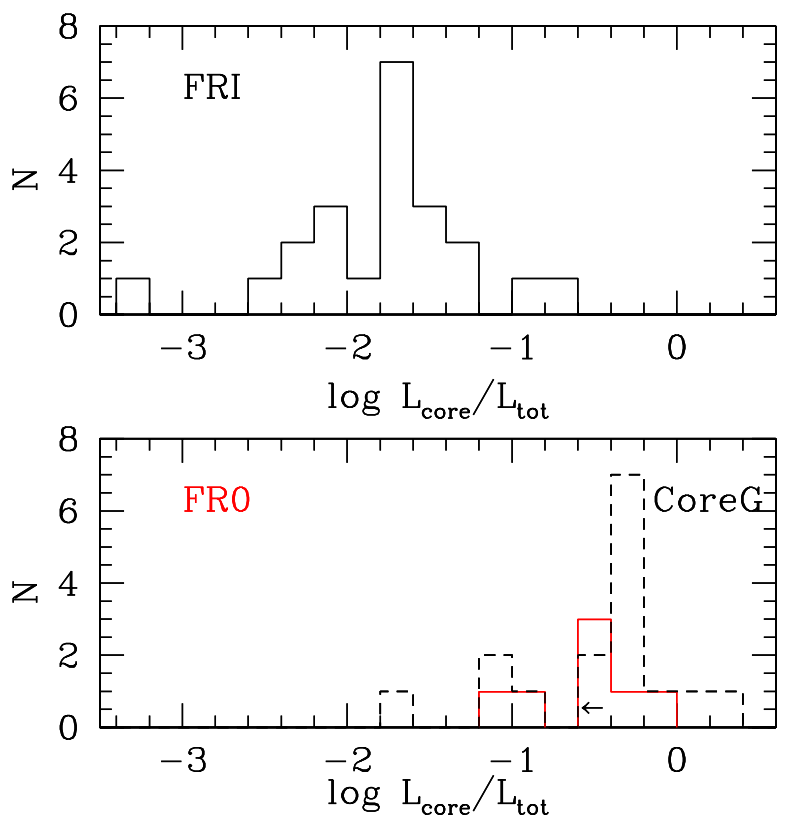

Fig. 7. Histograms of core dominance $\log R=\log \left(L_{\text {core }} / L_{\text {tot }}\right)$ for the $3 \mathrm{CR} / \mathrm{FR}$ Is (black solid line), CoreG (black dashed line), and our sample of seven FR 0s (red solid line).

are characterized by a core responsible for a fraction larger than $\gtrsim 10 \%$ of the total emission with structures extending for, at most, a few kpc. In classical FR Is and FR IIs RGs the core dominance is typically $\lesssim 1 \%$ and their sizes are of tens or hundreds of kpc (e.g Morganti et al. 1993, 1997). The high FR 0 core dominance appears to be due to the paucity of extended radio emission, rather than to an enhanced radio core, since FR 0s and FR I show similar ratios of radio-core to emission line luminosity. In addition, the FR 0 definition involves the photometric/spectroscopic nuclear and host properties (e.g., $\mathrm{BH}$ mass, color, and emission line ratios, similar to FR Is), which distinguish them from the radio sources associated with RQ AGN and star-forming galaxies.

This new class of RGs is similar to the low-luminosity radio sources hosted in ETGs, studied by Slee et al. (1994), which contain parsec-scale radio cores and do not produce extended radio emission. Furthermore, recently, Sadler et al. (2014) found that the bulk of the $20-\mathrm{GHz} \mathrm{RG}$ population consists of compact radio sources lacking of extended radio emission, analogous to our FR 0s.

The CoreG fulfill the requirements for a FR 0 classification: they show kpc scale radio structures and are of high core dominance, they are hosted in red giant ellipticals and are characterized by LEG line ratios. They are $\sim 100$ times less luminous than the FR 0s of the SDSS/NVSS sample discussed here. They smoothly extend the various nuclear multi-wavelengths relations seen in FR Is. In this sense, they represent the low-luminosity end of the FR 0 population that therefore extends at least down to a radio power of $\sim 10^{36} \mathrm{erg} \mathrm{s}^{-1}$.

The small size and the steep spectra of FR 0s might suggest the presence of compact steep spectrum (CSS) sources, that is, of rapidly growing young radio-sources (e.g., O'Dea 1998; Snellen et al. 2000). CSS show a correlation between the turnover frequency in their spectra and their linear sizes (e.g., Fanti et al. 1990; O'Dea 1998). The sizes of our FR 0 sources $(<3 \mathrm{kpc})$ does not contrast with their steep spectra since the turnover might occur at lower frequencies than the lowest value sampled by our observations, $1.4 \mathrm{GHz}$. However, the core dominance of CSS, although generally more difficult to measure and uncertain with respect to more extended radio-sources, is much lower than in FR 0s (typically $\log R \sim-1$ for quasars and $\log R \sim-2.4$ for galaxies Saikia et al. 1995, 2001), which rules out a general association of the FR 0s with the CSS.

As reported in the Introduction, the vast majority of the SDSS/NVSS objects fulfill the FR 0 definition, with the notable exception of the lack of a direct measurement of the core dominance, which is impossible from the FIRST images. Indeed, this was the main motivation for obtaining the higher resolution data presented in the previous sections. Nonetheless, the 
possibility that they are generally highly core dominated objects and thence bona-fide FR 0 appears to be reasonably founded. In fact, most of them $(\sim 80 \%)$ are unresolved or barely resolved in the FIRST maps, limiting their sizes to, at most, a few kpc. This is an indication that similarly to the seven objects studied, they have a deficit of extended radio emission with respect to classical extended RGs. It is clearly highly desirable to obtain proper radio data for a larger sample to base this conclusion on firmer ground. Therefore, if this result would be confirmed for a larger subsample of the SDSS/NSS RGs, the FR 0 population would be the dominant class of RGs in the local Universe with a space density $>100$ times higher than 3C sources.

FR 0s apparently differ from FR Is only for the lack of largescale radio structures. In the following we present two scenarios that can account for this specific property.

In the first scenario, the central engines of FR 0 s and FR Is are indistinguishable, and the paucity (and small size) of the extended radio emission is ascribed to an evolutionary effect. FR 0s might be young RGs that did not yet develop an extended radio structure. However, their number density should be smaller than that of FR Is, which is opposite to what is observed. This scenario can hold if FR 0s are intermittent sources. Rapid AGN intermittency (see, e.g., Readhead et al. 1994; Reynolds 1997; Czerny et al. 2009) would prevent FR 0s from becoming well-developed RGs. However, this does not explain why intermittency should affect only FR 0s, particularly considering the indications that accretion in FR Is and CoreG is associated with a long-lasting inflow of hot coronal gas (Allen et al. 2006; Balmaverde et al. 2008), which is only related to the host properties. Therefore, no differences would be expected between FR Os and FR Is.

As a second scenario, the differences between FR 0s and FR Is might be due to a lower jet bulk speed $\Gamma$ in FR 0s than in FR Is. In this scheme, the innermost regions of FR 0 and FR Is do not differ significantly since they share a common range of accretion rates (proved by the similarity in line emission luminosity and optical line ratios) and of radio core powers (which represent the synchrotron emission from the base of the jet). The different radio behavior should arise on a larger scale. In the hypothesis that jets in FR 0s are slower than FR Is, they are more subject to instabilities and entrainment (Bodo et al. 2013), which causes their premature disruption. Indeed, the typical scale of the radio emission in FR 0s is smaller than the core size of their hosts, a region characterized by a dense interstellar medium that obstructs the passage of the jet. This idea is supported, albeit with small number statistics, by the absence of one-sided kpc scale morphologies among the FR 0s observed, the typical sign of relativistic jet boosting. The ultimate origin of the lower $\Gamma$ factor in FR 0 is apparently not related to any directly observable quantity. We speculate that this could be due to a different spin of their central $\mathrm{BH}$, by assuming a dependence between the BH spin and $\Gamma$, as suggested by McKinney (2005), Tchekhovskoy et al. (2010), Chai et al. (2012), and Maraschi et al. (2012). The FR I radio morphology is only produced when the BH spin is close to its maximum value, while lower spin values could be associated with FR 0s. Recently, Ghisellini et al. (2014) predicted a population of jetted sources which have lower BH spin than classical RL AGN: the FR 0s might represent or be part of this population.

\section{Summary and conclusions}

We presented the observations of 12 objects with the JVLA in A-array configuration. These sources were selected from a large sample (cross-matching NVSS/FIRST and SDSS) that represents the bulk of the local radio-emitting AGN population (Baldi $\&$ Capetti 2010). Based on the FIRST radio maps, most of the sources are compact on an angular scale of $5^{\prime \prime}$.

The new high-resolution observations at 1.4, 4.5, and $7.5 \mathrm{GHz}$ revealed that these objects still show a compact morphology, extending at most over 1-3 kpc (with just one exception, a hybrid FR I/FR II which extends over $\sim 40 \mathrm{kpc}$ ). We isolated and measured the radio core component thanks either to the new high-resolution maps or to the radio spectra. Furthermore, the SDSS survey provides the spectro-photometric properties of the sample, such as BH mass, host type, emission lines. Based on these properties, we divided the sample into two groups.

The first group consists of four sources that represent the RQ AGN contamination to the SDSS/NVSS sample. They have low BH masses (mostly $\sim 10^{7} M_{\odot}$ ) associated with blue galaxies that show evidences of star formation.

The second group consists of seven RL AGN in red massive $\left(M_{*} \sim 10^{11} M_{\odot}\right.$ and BH masses $\gtrsim 10^{8} M_{\odot}$ ) ETGs spectroscopically classified as LEG. All these characteristics are shared by local FR Is. In particular, the members of this group have similar radio and [O III] line luminosities to those of FR Is, lying on the same relation; however, they show a core dominance higher than in FR Is by a factor $\sim 30$. This group is distinguished from FR Is by one single aspect: the lack of substantial extended emission. For this characteristic we called these objects "FR 0". This new RG class is consistent with a subclass of Gigahertz-Peaked Spectrum (GPS) radio sources proposed by Tingay \& Edwards (2015), with a low luminosity and with jet-dominated compact morphologies similar to FR Is and a lack of extended radio emission. Furthermore, recently, Sadler et al. (2014), studying the 20-GHz radio-source population in the local Universe, suggested that the FR 0s might be a mixed population of young CSS and GPS radio galaxies.

What causes the deficit of extended radio emission in FR 0s? We discussed two possible interpretations. The first scenario proposes that the FR 0s consist in young intermittent radio sources that will eventually evolve into extended RGs. However, this contrasts with the picture in which low-luminosity RGs are powered by continuous accretion of hot gas from their X-ray coronae. Furthermore, it does not explain why the intermittency should only occur in FR Os and not in FR Is, considering the similarity of their nuclear and host properties.

The second scenario suggests that FR 0s have smaller jet Lorentz factors $\Gamma$ than FR Is: their jets are less stable against entrainment, and their passage through the dense ISM region of the central regions of their hosts causes their premature disruption. This low- $\Gamma$ scenario can be tested by examining the asymmetry between the opposite jets in FR Os, a parameter directly linked to the jet speed. Although twin symmetric jets are common in FR Is, they often have one jet substantially brighter than the other on kpc scale (e.g., Parma et al. 1987). This indicates that the high Doppler factors derived for FR Is on pc scale (based on the detection of superluminal motions, e.g., Giovannini et al. 2001, and, indirectly, on the association with BL Lac objects in the AGN unified model, e.g., Urry \& Padovani 1995) are preserved to larger scales. In the present sample we found that all three FR 0s with an extended radio structure are two-sided and rather symmetric. The number is too small to draw any firm conclusion (and two-sided jets are expected to be more common than a one-sided morphology in a randomly oriented sample) but it is consistent with this hypothesis. We speculate that the ultimate difference between FR Os and FR Is is the spin of their $\mathrm{BH}$, which is smaller for FR 0s. 

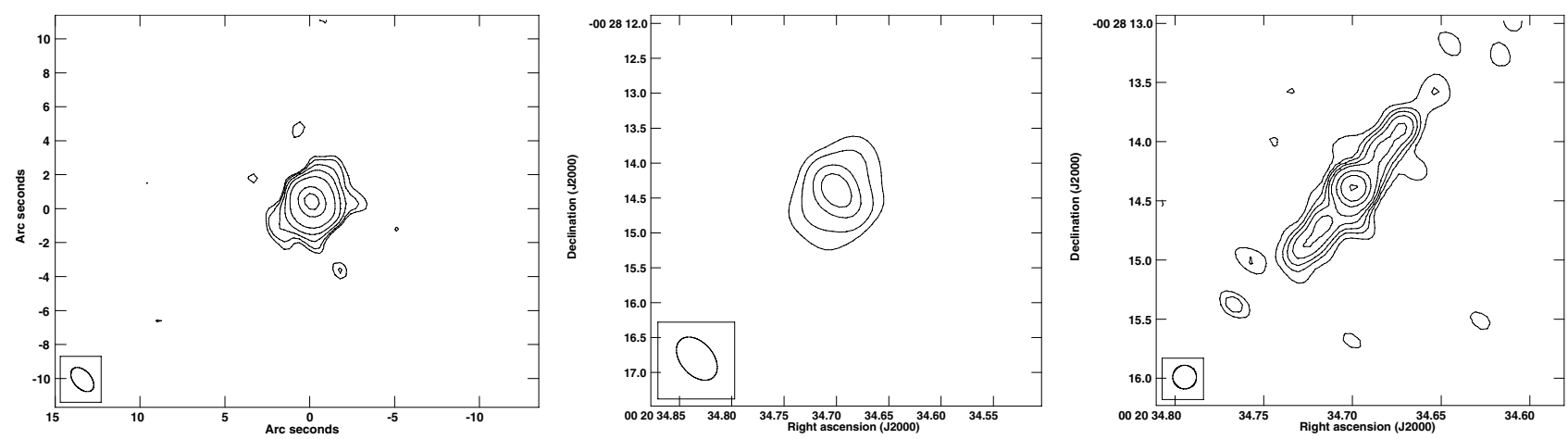

Fig. A.1. JVLA images of ID 547 at 1.4, 4.5 and $7.5 \mathrm{GHz}$. The HPBWs and noise levels are reported in Table 2 . Levels are 0.150 .20 .30 .513 $5 \mathrm{mJy} /$ beam; $0.30 .50 .91 .3 \mathrm{mJy} /$ beam; $0.030 .050 .070 .10 .150 .30 .50 .9 \mathrm{mJy} /$ beam.
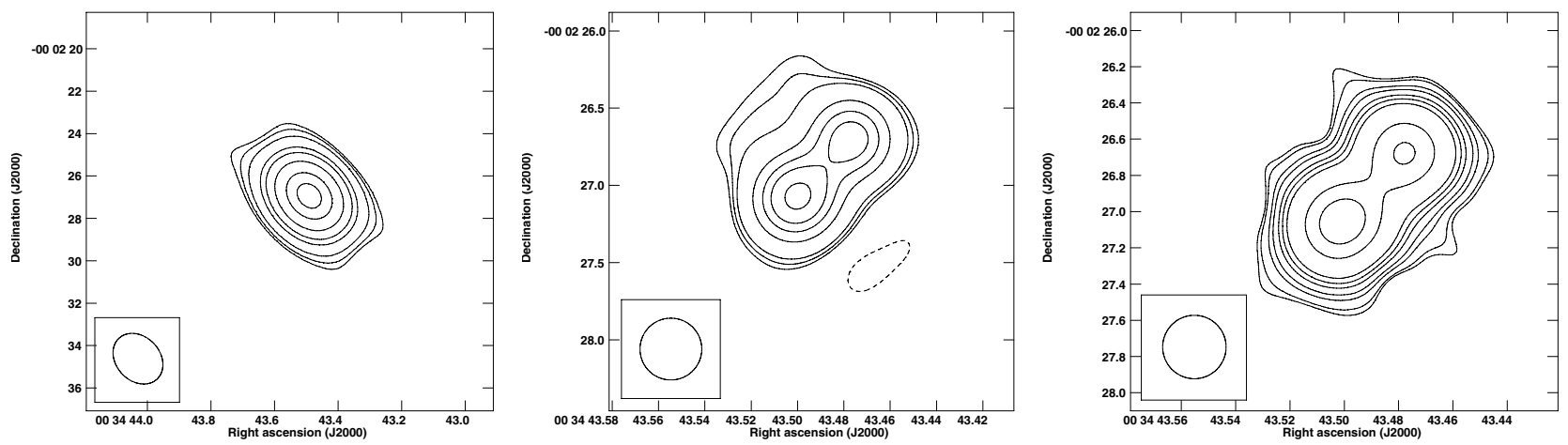

Fig. A.2. JVLA images of ID 568 at 1.4, 4.5 and $7.5 \mathrm{GHz}$. The HPBWs and noise levels are reported in Table 2. Levels are 0.30 .51351020 $30 \mathrm{mJy} /$ beam; -0.5 0.5 0.713571020305070 mJy/beam; $0.070 .10 .20 .30 .50 .7135 \mathrm{mJy} /$ beam.
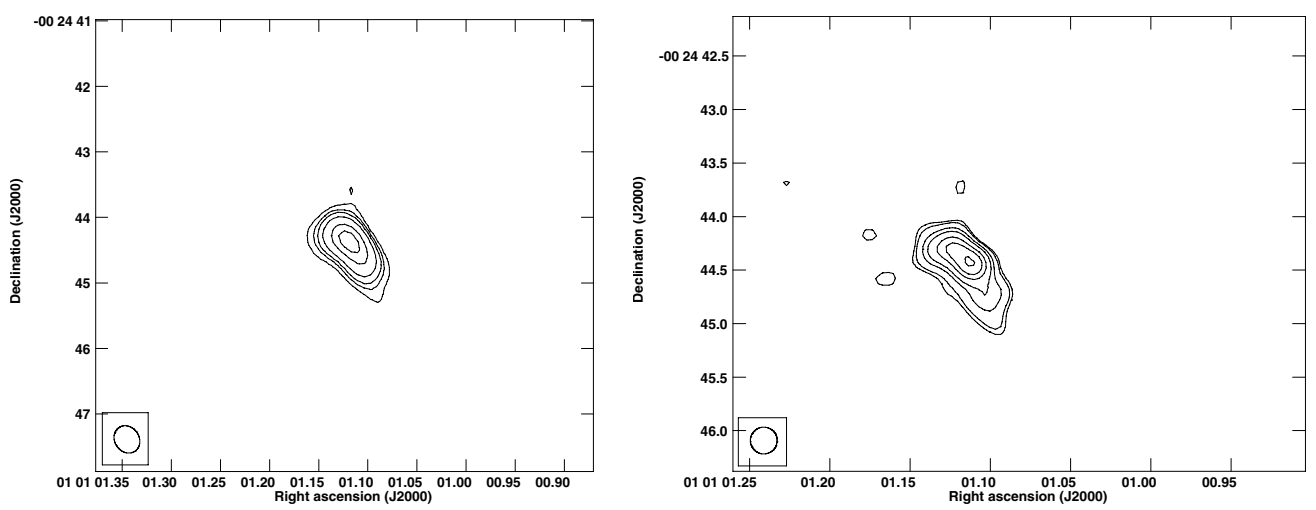

Fig. A.3. JVLA images of ID 590 at 4.5 and $7.5 \mathrm{GHz}$. The HPBWs and noise levels are reported in Table 2 . Levels are 0.10 .20 .30 .51 $1.5 \mathrm{mJy} /$ beam; $0.070 .10 .20 .30 .50 .71 \mathrm{mJy} /$ beam.

High-resolution imaging of a larger sample of local lowluminosity radio sources is needed to put this result on firmer statistical ground and confirm the presence of the FR 0 population. To improve the efficiency of such a survey, the results obtained in this study indicate that it should be focused on objects with a LEG spectrum, hosted in red early type galaxies. If the FR 0 population were confirmed, they would represent the bulk of the RL AGN in the local Universe with a strong impact on our view of the low-luminosity radio-emitting population.

\section{Appendix A: Notes on the extended radio-sources}

We report here notes on the structure of the extended radiosources in the sample.

ID 547: while at 1.4 and $4.5 \mathrm{GHz}$ it is only marginally resolved, a clear twin-jet morphology is revealed at the higher frequency (Fig. A.1). The two jets are rather straight and symmetric, extending for about $1^{\prime \prime}$ on each side of the nucleus along $\mathrm{PA} \sim-40^{\circ}$.

ID 568: its radio morphology is double ( $\sim 0^{\prime} 5$ in size) with $\mathrm{PA}=\sim 130^{\circ}$ (Fig. A.2). The position of the two lobes are $\left(00^{\mathrm{h}} 34^{\prime} 43^{\prime \prime} .51-00^{\mathrm{d}} 02^{\prime} \quad 27^{\prime \prime} .06\right)$ and $\left(00^{\mathrm{h}} 34^{\prime} \quad 43^{\prime \prime} .48\right.$ $-00^{\mathrm{d}} 02^{\prime} 26^{\prime \prime}$.67), whose fluxes at 4.5 and 7.5 are 15.3 and $6.7 \mathrm{mJy}$ for the former component and 8.4 and $5.3 \mathrm{mJy}$ for the latter. The resolution is insufficient to resolve substructures or the presence of a radio core.

ID 590: the source is point-like in $L$ band, while at high frequencies the source is elongated with PA $=45^{\circ}$ with a size of $0 .^{\prime} 8$ (Fig. A.3) with a morphology suggestive of a bent two-sided jet structure.

ID 625: this source shows a central emission and a double structure in the NS direction (Fig. A.4) on a scale of $36^{\prime \prime}, \sim 40 \mathrm{kpc}$ 
R. D. Baldi et al.: Pilot study of the radio-emitting AGN population: the emerging new class of FR 0 radio-galaxies
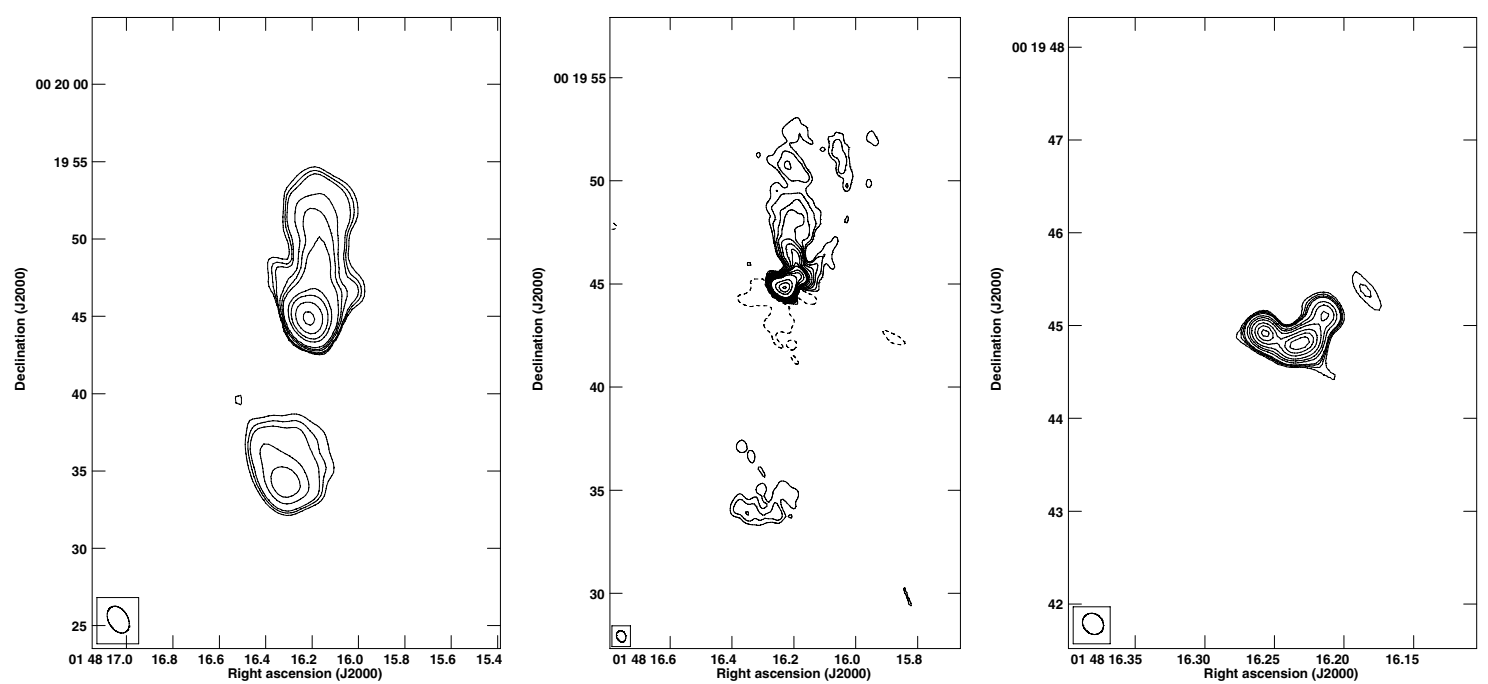

Fig. A.4. JVLA images of ID 625 at 1.4, 4.5 and 7.5 GHz. The HPBWs and noise levels are reported in Table 2. Levels are 22.5357103050 $100200 \mathrm{mJy} /$ beam; -0.2 0.2 0.3 0.5 0.7 11.522.535710305070 mJy/beam; $1.522 .535710152025 \mathrm{mJy} /$ beam.

in low resolution map. Toward the south only a diffuse lobe is visible, while toward the north there is a bright jet-like structure, typical of FR Is, terminating into a diffuse plume. This radio structure is typical of the so-called hybrid radio-sources (Gopal-Krishna \& Wiita 2000). At high resolution the nuclear source is resolved and rather complex. A compact flat spectrum region (located at $01^{\mathrm{h}} 48^{\prime} 16^{\prime \prime} \cdot 25-00^{\mathrm{d}} 19^{\prime} 44^{\prime \prime} \cdot 9$ ) is identified as the radio core based on its flat spectral index $(0.05$ between 4.5 and $7.5 \mathrm{GHz}$ ). From the core a one-sided emission is visible, pointing SW, but it sharply bends toward the NE after $\sim 2^{\prime \prime}$ from the core and then enters the $\mathrm{N}$ lobe. Two knots are visible in the $7.5 \mathrm{GHz}$ image with spectral index 0.6 and 1.16 respectively. The strong jet bending and the jet asymmetry suggest that a relativistic jet at a small angle with respect to the line of sight is present in this source.

Acknowledgements. R.D.B. was supported at the Technion by a fellowship from the the Lady Davis Foundation. We thank the anonymous referee for the constructive comments that have helped us to improve the paper.

\section{References}

Allen, S. W., Dunn, R. J. H., Fabian, A. C., Taylor, G. B., \& Reynolds, C. S. 2006, MNRAS, 372, 21

Baldi, R. D., \& Capetti, A. 2008, A\&A, 489, 989

Baldi, R. D., \& Capetti, A. 2009, A\&A, 508, 603

Baldi, R. D., \& Capetti, A. 2010, A\&A, 519, A48

Baldi, R. D., Chiaberge, M., Capetti, A., et al. 2010, ApJ, 725, 2426

Baldwin, J. A., Phillips, M. M., \& Terlevich, R. 1981, PASP, 93, 5

Balmaverde, B., \& Capetti, A. 2006, A\&A, 447, 97

Balmaverde, B., Capetti, A., \& Grandi, P. 2006, A\&A, 451, 35

Balmaverde, B., Baldi, R. D., \& Capetti, A. 2008, A\&A, 486, 119

Balogh, M. L., Morris, S. L., Yee, H. K. C., Carlberg, R. G., \& Ellingson, E. 1999, ApJ, 527, 54

Bell, E. F., McIntosh, D. H., Katz, N., \& Weinberg, M. D. 2003, ApJS, 149, 289

Bell, E. F., Zheng, X. Z., Papovich, C., et al. 2007, ApJ, 663, 834

Bernardi, M., Shankar, F., Hyde, J. B., et al. 2010, MNRAS, 404, 2087

Best, P. N., \& Heckman, T. M. 2012, MNRAS, 421, 1569

Best, P. N., Kauffmann, G., Heckman, T. M., \& Ivezić, Ž. 2005, MNRAS, 362 , 9

Bodo, G., Mamatsashvili, G., Rossi, P., \& Mignone, A. 2013, MNRAS, 434, 3030

Buttiglione, S., Capetti, A., Celotti, A., et al. 2010, A\&A, 509, A6

Cao, X, \& Rawlings, S. 2004, MNRAS, 349, 1419

Capetti, A., \& Baldi, R. D. 2011, A\&A, 529, A126
Chai, B., Cao, X., \& Gu, M. 2012, ApJ, 759, 114

Chiaberge, M., \& Marconi, A. 2011, MNRAS, 416, 917

Chiaberge, M., Capetti, A., \& Celotti, A. 1999, A\&A, 349, 77

Chiaberge, M., Capetti, A., \& Celotti, A. 2002, A\&A, 394, 791

Czerny, B., Siemiginowska, A., Janiuk, A., Nikiel-Wroczyński, B., \& Stawarz, Ł. 2009, ApJ, 698, 840

Edelson, R. A. 1987, ApJ, 313, 651

Fanaroff, B. L., \& Riley, J. M. 1974, MNRAS, 167, 31P

Fanti, R., Fanti, C., Schilizzi, R. T., et al. 1990, A\&A, 231, 333

Floyd, D. J. E., Axon, D., Baum, S., et al. 2008, ApJS, 177, 148

Ghisellini, G., Tavecchio, F., Maraschi, L., Celotti, A., \& Sbarrato, T. 2014, Nature, 515, 376

Giovannini, G., Cotton, W. D., Feretti, L., Lara, L., \& Venturi, T. 2001, ApJ, 552, 508

Gopal-Krishna \& Wiita, P. J. 2000, A\&A, 363, 507

Heckman, T. M. 1980, A\&A, 87, 152

Kauffmann, G., Heckman, T. M., White, S. D. M., et al. 2003, MNRAS, 341, 33

Kewley, L. J., Groves, B., Kauffmann, G., \& Heckman, T. 2006, MNRAS, 372, 961

Maraschi, L., Colpi, M., Ghisellini, G., Perego, A., \& Tavecchio, F. 2012, J. Phys. Conf. Ser., 355, 012016

McKinney, J. C. 2005, ApJ, 630, L5

Morganti, R., Killeen, N. E. B., \& Tadhunter, C. N. 1993, MNRAS, 263, 1023

Morganti, R., Oosterloo, T. A., Reynolds, J. E., Tadhunter, C. N., \& Migenes, V. 1997, MNRAS, 284, 541

Nagar, N. M., Falcke, H., \& Wilson, A. S. 2005, A\&A, 435, 521

Nakamura, O., Fukugita, M., Yasuda, N., et al. 2003, AJ, 125, 1682

O’Dea, C. P. 1998, PASP, 110, 493

Parma, P., Fanti, C., Fanti, R., Morganti, R., \& de Ruiter, H. R. 1987, A\&A, 181, 244

Readhead, A. C. S., Xu, W., Pearson, T. J., Wilkinson, P. N., \& Polatidis, A. G. 1994, in Compact Extragalactic Radio Sources, eds. J. A. Zensus, \& K. I. Kellermann, 17

Reynolds, C. S. 1997, MNRAS, 286, 513

Sadler, E. M., Ekers, R. D., Mahony, E. K., Mauch, T., \& Murphy, T. 2014, MNRAS, 438, 796

Saikia, D. J., Jeyakumar, S., Wiita, P. J., Sanghera, H. S., \& Spencer, R. E. 1995, MNRAS, 276, 1215

Saikia, D. J., Jeyakumar, S., Salter, C. J., et al. 2001, MNRAS, 321, 37

Shen, S., Mo, H. J., White, S. D. M., et al. 2003, MNRAS, 343, 978

Slee, O. B., Sadler, E. M., Reynolds, J. E., \& Ekers, R. D. 1994, MNRAS, 269, 928

Snellen, I. A. G., Schilizzi, R. T., Miley, G. K., et al. 2000, MNRAS, 319, 445

Strateva, I., Ivezić, Ž., Knapp, G. R., et al. 2001, AJ, 122, 1861

Tchekhovskoy, A., Narayan, R., \& McKinney, J. C. 2010, ApJ, 711, 50

Tingay, S. J., \& Edwards, P. G. 2015, MNRAS, in press [arXiv: 1501.04393]

Tremaine, S., Gebhardt, K., Bender, R., et al. 2002, ApJ, 574, 740

Urry, C. M., \& Padovani, P. 1995, PASP, 107, 803

Zirbel, E. L. 1996, ApJ, 473, 713 